\title{
Strategies for fast and low-dose laboratory-based phase contrast tomography for microstructural scaffold analysis in tissue engineering
}

Charlotte K. Hagen ${ }^{\mathrm{a}}$, Panagiotis Maghsoudlou ${ }^{\mathrm{b}}$, Giorgia Totonellic, Paul C. Diemoz ${ }^{\mathrm{a}}$, Marco Endrizzi $^{\mathrm{a}}$, Anna Zamir ${ }^{\mathrm{a}}$, Paola Coan ${ }^{\mathrm{d}, \mathrm{e}}$, Alberto Bravin ${ }^{\mathrm{f}}$, Paolo De Coppi ${ }^{\mathrm{b}}$, and Alessandro Olivo $^{\text {a }}$

${ }^{a}$ Department of Medical Physics and Biomedical Engineering, University College London, Malet Place, London WC1E 6BT, United Kingdom

${ }^{b}$ Institute of Child Health, University College London, Guilford Street, London WC1N 1EH, United Kingdom

'Bambino Gesu Children's Hospital, Piazza di Sant'Onofrio 4, 00165 Rome, Italy

${ }^{\mathrm{d}}$ Faculty of Medicine, Ludwig Maximilians University, Marchioninistrasse 15D, 81377

Grosshadern-Munich, Germany

eDepartment of Physics, Ludwig Maximilians University, Am Coulombwall 1, 85748 Garching, Germany

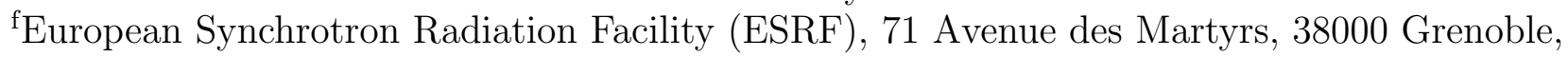
France

\begin{abstract}
The application of x-ray phase contrast computed tomography (PCT) to the field of tissue engineering is discussed. Specific focus is on the edge illumination PCT method, which can be adapted to weakly coherent x-ray sources, permitting PCT imaging in standard (non-synchrotron) laboratory environments. The method was applied to a prominent research topic in tissue engineering, namely the development of effective and reliable decellularization protocols to derive scaffolds from native tissue. Results show that edge illumination PCT provides sufficient image quality to evaluate the microstructural integrity of scaffolds and, thus, to assess the performance of the used decellularization technique. In order to highlight that edge illumination PCT can ultimately comply with demands on a high specimen throughput and low doses of radiation, recently developed strategies for scan time and dose reduction are discussed.
\end{abstract}

Keywords: Tissue Engineering, Decellularization, X-Ray Phase Contrast, Computed Tomography

\section{INTRODUCTION}

The demand for organ transplantation has rapidly increased during the past decade. At the same time, a severe donor shortage and the likely rejection of transplant organs by the recipient prevent this demand from being met. Tissue engineering provides a potential solution as it aims to develop transplant organs by combining appropriate scaffolds and cells. An important research question is how to produce suitable scaffolds; evidence suggests that the scaffold microstructure, often considered a surrogate marker for biomechanical properties, and extracellular matrix composition are important factors. ${ }^{1}$ Scaffold development can be classified into "synthetic" approaches, which exploit 3D-printing or moulding techniques to produce scaffolds from biodegradable polymers, and "natural" approaches, which derive scaffolds from cadaver tissue through decellularization protocols based on detergents and enzymes. The latter approach is often considered favourable for complex tissue due to the presence of native microstructure, biomechanical properties and matrix composition. ${ }^{2}$

Send correspondence to CKH, E-mail: charlotte.hagen.10@ucl.ac.uk

Developments in X-Ray Tomography X, edited by Stuart R. Stock, Bert Müller, Ge Wang, Proc. of SPIE Vol. 9967, 996705 - (c) 2016 SPIE' CCC code: 0277-786X/16/\$18 · doi: 10.1117/12.2237594

Proc. of SPIE Vol. 9967 996705-1 
The development of effective and reliable tissue decellularization protocols is an active area of research. Current work concentrates on developing "gentle" methods that remove all cellular material whilst maintaining the matrix microstructure. ${ }^{3}$ To aid their development, and, more generally, to establish criteria for successful scaffold production, imaging of scaffolds after decellularization is needed. At present, electron microscopy is the standard analytic technique; however, it requires a destructive sample preparation; therefore, it is not suitable for volumetric analyses. Optical techniques are inappropriate due to the mandatory sample slicing/staining, as well as due to their limited penetration depth in tissue. The applicability of established 3D imaging modalities like magnetic resonance imaging (MRI) or x-ray-based micro-CT is also limited; MRI struggles to achieve the required spatial resolution (ideally micrometres) within feasible scan times, and micro-CT fails to achieve sufficient soft tissue contrast.

On the contrary, x-ray phase contrast tomography (PCT) has the potential to overcome these issues as it has a high penetration depth, is intrinsically a 3D imaging method, and can provide increased soft-tissue contrast. ${ }^{4}$ The latter stems from the exploitation of phase effects, which are larger than attenuation ones within the diagnostic energy range and for materials with a small linear attenuation coefficient. ${ }^{5}$ When appropriately processed, PCT images are quantitative and show the refractive index distribution within the sample. ${ }^{6}$ Over the past two decades, PCT has been the focus of extensive research by various groups around the world, a major milestone being the adaptation to non-synchrotron, weakly coherent x-ray sources. ${ }^{7,8}$ This has simplified access to the technology and, thus, expanded its applicability to new areas, e.g. the rapidly growing field of tissue engineering.

In the following, the focus is on a specific PCT method (edge illumination) and its potential to be used for scaffold analysis following decellularization. The method has recently been applied to natural scaffolds derived from a range of complex organs, revealing a high image quality. ${ }^{9}$ However, that study has concentrated primarily on synchrotron-based imaging, with only a minor focus on the potential for a translation into standard laboratory environments. In the following, a more in-depth discussion of the topic is provided. After a brief introduction to the method, results are presented which demonstrate that information relevant to the evaluation of decellularization protocols can be obtained. Finally, new imaging concepts currently under development and aimed at scan time and dose reduction are discussed.

\section{EDGE ILLUMINATION X-RAY PHASE CONTRAST COMPUTED TOMOGRAPHY}

The edge illumination method has initially been developed for planar imaging at synchrotrons, ${ }^{10}$ where thin laminar beams are available. By aligning a laminar beam with the edge of a row of pixels, sensitivity to sample refraction (i.e. the macroscopic manifestation of the phase shift) is created, as the small deviation of x-rays induced by the sample causes an increased or decreased beam portion to hit the pixels and be detected. Unlike other x-ray phase contrast techniques, the edge illumination method is not based on interference; thus, it has relaxed coherence requirements and can be adapted to non-microfocal laboratory sources. ${ }^{8}$ In order to fully

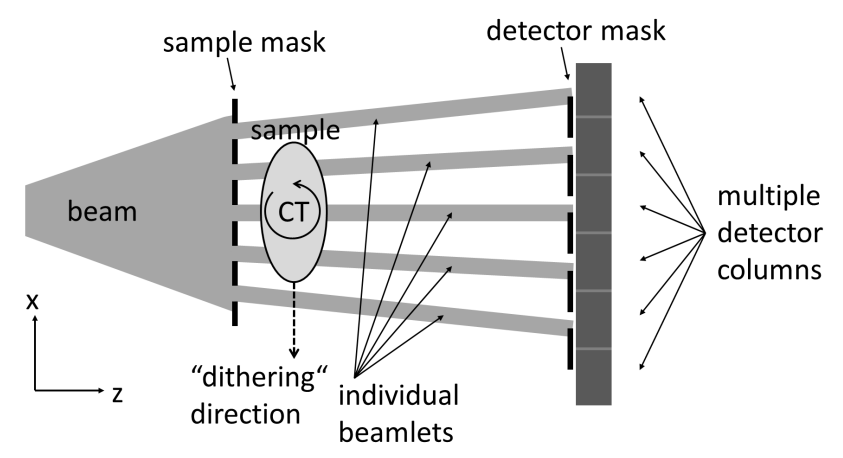

Figure 1. Schematic of a full-field edge illumination x-ray phase contrast tomography setup (seen from top). 
exploit the cone beams produced by such sources, two masks are added to the setup. The first one ("sample mask") is positioned immediately upstream the sample and sections the cone beam into an array of beamlets, which are separated by a distance large enough to prevent them from interfering. The second one ("detector mask") is positioned in front of the detector and creates insensitive regions between pixel rows or columns. By introducing a relative shift between the two masks through a slight offset of the sample mask, each beamlet impinges on an "edge" of the detector mask, which effectively replicates the synchrotron configuration across an extended field of view. This full-field implementation of the edge illumination method is schematically shown in Figure 1.

In order to quantify sample refraction, this must be separated from attenuation, with which it is inevitably entangled. This requires a second image to be taken after introducing a sample mask offset in the opposite direction, such that the beamlets impinge on opposite "edges" of the detector mask in the two cases. The application of adequate data processing methods (so-called "phase retrieval") ${ }^{11,12}$ then provides a refraction angle image, which is quantitatively described by: $\alpha(x)=d / d x \int \delta(x, z) d s$. Here, $\delta$ is the refractive index of the sample, and the integration extends along the direction of beam propagation.

The extracted refraction angle image features a line integral relationship between measurements and the sample refractive index, allowing tomographic principles to be applied. Thus, 3D images ("tomograms") can be obtained after data is collected at many rotational sample views covering a range of at least 180 degrees. Image reconstruction can be performed via standard methods, e.g. filtered back projection (FBP); however, since the refraction angle is a differential quantity, a special filter function (Hilbert filter) must be used.

\section{APPLICATION TO TISSUE ENGINEERING: STRUCTURAL ANALYSIS OF DECELLULARIZED TISSUE}

Edge illumination PCT is thus a means to obtain 3D images of weakly attenuating samples with improved image contrast. To demonstrate the benefit for the field of tissue engineering, a natural scaffold derived from a rabbit esophagus via the "detergent enzymatic treatment" (DET) decellularization protocol ${ }^{13}$ was scanned with a laboratory-based imaging setup. To enable a structural comparison, a control specimen was also scanned; this was a rabbit esophagus which was not decellularized, but which otherwise underwent the same sample preparation as the scaffold, namely fixation in $2.5 \%$ glutaraldehyde in $0.1 \mathrm{M}$ phosphate buffer $(\mathrm{pH} 7.4)$ and critical point drying using $\mathrm{CO}_{2}$. For imaging, the samples were placed in a plastic cylinder.

The used experimental setup is described in detail elsewhere, ${ }^{9}$ but it should be highlighted here that it was comprised entirely of commercially available equipment. The x-ray tube was a Rigaku MicroMax 007 HF x-ray tube with rotating molybdenum target and a focal spot with a horizontal dimension of approximately $70 \mu \mathrm{m}$
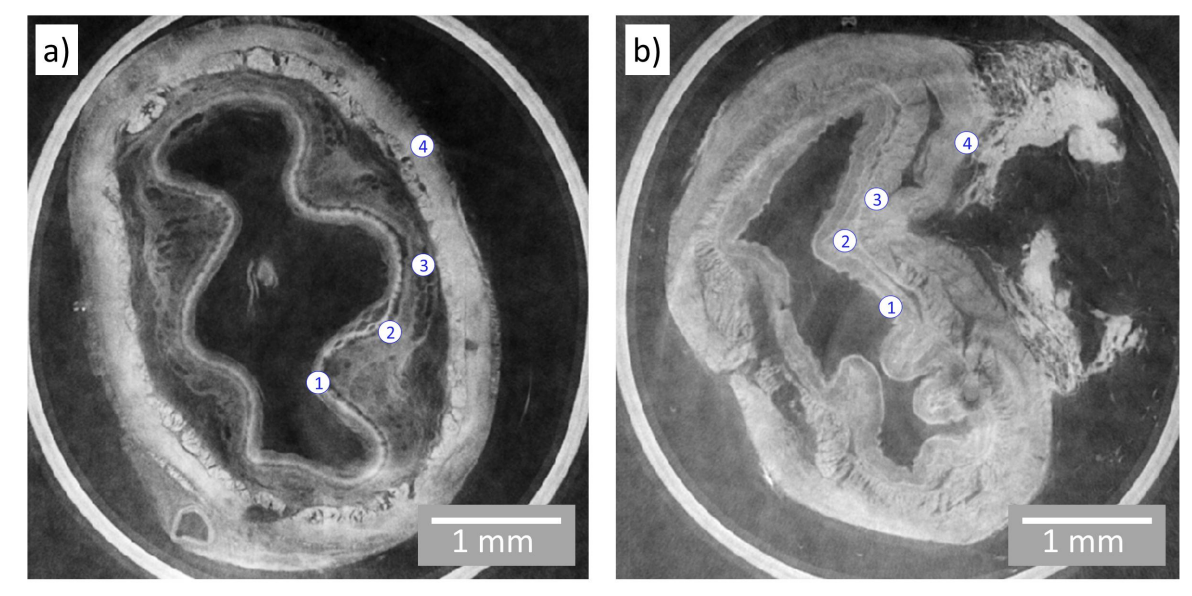

Figure 2. Edge illumination x-ray phase tomograms showing a decellularized (a) and non-decellularized (b) rabbit esophagus. The markers indicate the mucosa (1), sub-mucosa (2), muscularis propria (3) and adventitia (4). 
(full width half maximum), operated at $25 \mathrm{~mA}$ and $40 \mathrm{keV}$ (without any additional filtration), and the detector was a Hamamatsu C9732DK flat panel, a passive-pixel CMOS sensor with a pixel size of 50 x $50 \mu \mathrm{m}^{2}$. Data were collected for 360 equally spaced rotational views of the sample over a total angular range of 180 degrees. At each rotational view, two images were taken with inverted sample mask offsets to enable the quantitative extraction of refraction angle images prior to tomographic reconstruction. At each rotation angle, the sample was also scanned with ten sub-pixel steps; data were collected at each step and successively combined. This procedure, often referred to as "dithering", allows increasing the spatial resolution beyond what is dictated by the demagnified pixel size. ${ }^{14}$ Each frame was acquired with an integration time of $1.2 \mathrm{~s}$. Tomograms were reconstructed via FPB with the Hilbert filter.

The results for the scaffold and the non-decellularized control specimen are shown in Figure 2. Notably, all native anatomical layers of the esophagus (mucosa, sub-mucosa, muscularis propria, adventitia) can be detected in both images, providing confirmation of their presence and integrity within the scaffold. Intact blood vessels were detected in the submucosa and a clear demarcation was seen between the inner circular and outer longitudinal layers of the muscularis propria. The mucosal-submucosal separation, a surrogate marker for decellularizationassociated damage, could be detected across the entire circumference of a long segment of the scaffold, and was shown to be minimal.

This insight provides immediate valuable feedback on the applied decellularization protocol (DET), both in terms of its effectiveness and reliability, considering that a major challenge in the development of decellularization protocols is the removal of all cellular material while maintaining the native scaffold architecture.

\section{CONCEPTS FOR SCAN TIME AND DOSE REDUCTION}

The above results imply a strong potential of laboratory-based edge illumination PCT to benefit the field of tissue engineering. However, the ultimate impact and widespread use within this discipline depends on the achievable specimen throughput, as typically in biomedical research large sample numbers are encountered. A second success-defining aspect is dosimetry; while less important for the here presented analysis of cell-free scaffolds, for future investigations of cell behaviour on scaffolds, or for monitoring scaffolds post-implantation dose must be considered. Taking into account that reductions of scan time and dose typically come at the cost of a reduced image quality, the aim must be to minimize these parameters whilst maintaining an image quality that provides relevant analytical information. In the following, a review of currently developed imaging concepts for scan time and dose reduction is provided.

Until now, edge illumination PCT scans have been rather lengthy; for high statistic and high resolution imaging parameters (long integration times, several dithering steps) scan times of hours are not uncommon. ${ }^{15}$
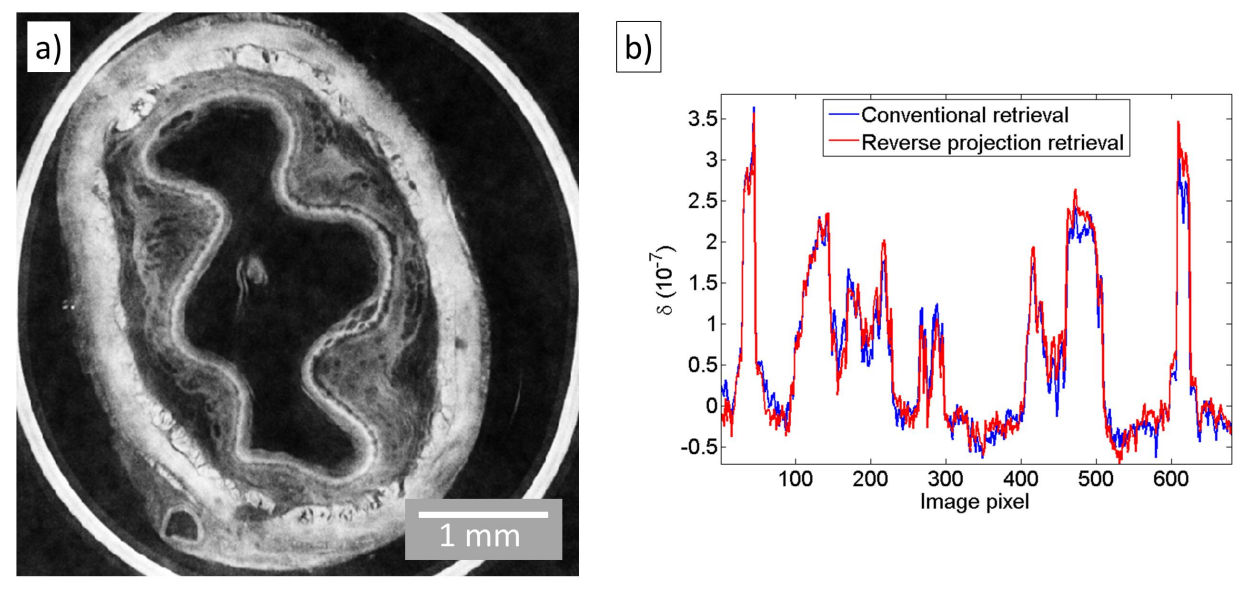

Figure 3. a) Edge illumination x-ray phase contrast tomogram of a decellularized rabbit esophagus, processed with the reverse projection method, b) Profile plots extracted horizontally across a central region of the reverse projection image (red curve) and across the image shown in Figure 2(a) (blue curve). 
This is primarily due to the flux limitation of non-synchrotron x-ray sources, especially when collimated by the sample mask. However, it is also due to the fact that two images are needed to extract refraction angle images prior to tomographic reconstruction. As discussed above, these images are taken at inverted offsets of the sample mask. In practice, the sample mask has to be moved at every rotational sample view, imposing an "interruption" of the acquisition which causes excessive overheads in addition to the detector integration time.

To overcome this issue, an alternative imaging concept ("reverse projection") has been developed. The reverse projection method had originally been devised for different PCT methods, ${ }^{16,17}$ but has recently been adapted to edge illumination data. ${ }^{18}$ In brief, it exploits that two images acquired with a sample rotation offset of 180 degrees between them provide the same information as two images acquired with inverted sample mask offsets, allowing the extraction of the refraction angle from these data. As the physical movement of the sample mask at each rotational view is no longer necessary, the overheads caused by the mask shifts are eliminated, the only requirement being that data are acquired for a 360 degree sample rotation instead of a 180 degree one. This significantly simplifies the experimental procedure and enables more efficient (and thus faster) scans. A detailed description of the reverse projection method is provided elsewhere. ${ }^{18}$

Figure 3 shows the result of applying the reverse projection concept to the scaffold data shown before. This is the same transverse sample slice as in Figure 2(a), but now retrieved from images taken over a range of 360 instead of 180 degrees, at one offset position of the sample mask only. All other scan and reconstruction parameters remained the same. The tomogram has the same visual appearance as the one shown in Figure 2(a), which is quantitatively confirmed by profile plots extracted horizontally across the central region of the scaffold (Figure 3(b)). This agreement demonstrates that reverse projection retrieval is indeed a reliable means to extract the refraction angle image.

In this example, dithering, i.e. the acquisition of multiple images for sub-pixel sample shifts per view angle was applied, implying that effectively only the overheads caused by the sample mask movement are eliminated, while those caused by the dithering itself are still present. Note however that these data should be regarded as proof-of-principle for the applicability of reverse projection retrieval. Generally, when dithering is not needed (i.e. the spatial resolution provided by the demagnified pixel size is sufficient to resolve features of interest), the remaining overheads are also removed. Tomographic scans can then be realized with a continuous (rather than stepped and interrupted) sample rotation, implying that the overall scan time is a function only of the total detector integration time (plus a small contribution by the read-out).

X-ray phase contrast imaging has been shown in several instances to have low-dose imaging capabilities. ${ }^{19,20}$ This is often attributed to the fact that the refractive index decreases slower with energy than the linear attenuation coefficient (which is exploited in conventional x-ray imaging), thus permitting the use of higher x-ray energies, which reduces dose deposition. Nevertheless, the dose delivered during a PCT scan ultimately depends also on the exposure time per frame and the number of frames acquired. While edge illumination PCT can benefit from the use of higher energies, until now it has relied on the acquisition of a large number of frames. Specifically, the need of two frames to extract the refraction angle is a drawback. Although the above discussed concept of reverse projection eliminates overheads caused by the acquisition of these two frames, it does not reduce the overall number of images (data must be collected over 360 instead of 180 degrees). However, a new imaging concept has recently been developed which eliminates the need of a second frame altogether. ${ }^{21}$ This so-called "single-shot" method is based on the assumption of a single-material object and small refraction angles, which are reasonable approximations for quasi-homogenous, soft tissue-based samples like naturally derived scaffolds. In brief, the method derives a relationship between edge illumination images and the thickness of the sample, which can be inverted via a Fourier filtering operation. This enables the retrieval of the thickness and, as this can be written as a line integral across the sample, tomographic reconstruction. Data demonstrating the single-shot imaging concept are not shown in this document, but can be found elsewhere. ${ }^{21}$

Single-shot imaging is thus a means to halve the overall number of frames, implying a potential dose reduction by a factor of two. However, future studies are needed to determine whether the smaller data volume must be balanced by a correspondingly increased photon statistics to ensure that image quality is identical as in previous methods. 


\section{SUMMARY AND DISCUSSION}

The potential of laboratory-based PCT for imaging applications in tissue engineering has been discussed. The specific focus was on the edge illumination PCT method and on the imaging of natural scaffolds obtained via decellularization, which is needed to evaluate the applied decellularization protocol. The presented results, i.e. tomograms of a decellularized esophagus and a non-decellularized control specimen, show high image quality, especially with regards to contrast and detail visibility. Notably, anatomical features such as the mucosalsubmucosal separation in the esophageal scaffolds could be identified. Native scaffold architecture was clearly visible and intact, allowing an assessment of the decellularization method used.

Besides these results, a new imaging concept enabling faster acquisitions via the elimination of overheads was discussed and applied to the scaffold data. It was highlighted that for samples for which the spatial resolution provided by the detector pixel size is sufficient, a continuous sample rotation can be performed. In such cases, scan time is determined only by the detector integration time, but is no longer constrained by physical translations of the sample mask or the sample itself.

Overall, the data shown can be considered additional key evidence for the potential benefit of PCT to the field of tissue engineering. A crucial difference between PCT and the gold-standard method currently used by the tissue engineering community to analyse scaffold microstructure (electron microscopy) is that it is nondestructive, which makes it a long-sought solution for imaging scaffolds prior to transplantation. While the above had previously been demonstrated through synchrotron experiments, the ability to perform scans in standard research laboratories could be the key step towards the practical use of PCT. For example, PCT could, already in the short term, become a tool for routine quality control of scaffolds produced via decellularization.

Longer-term goals could include the use of PCT for monitoring cell-scaffold interactions or scaffold behaviour and functionality in vivo after transplantation into live organisms, e.g. mice. Such applications will require further studies, primarily into the effect of radiation dose on cell fate. In this context, it should be noted that x-ray phase contrast imaging has been shown in several instances to have low-dose imaging capabilities. ${ }^{19,20}$ Furthermore, as discussed above, the recently developed concept of single- shot imaging holds promise for further dose reduction.

\section{ACKNOWLEDGMENTS}

This work was supported by the UK Engineering and Physical Sciences Research Council (Grant Nos. EP/L001381/1 and EP/I021884/1). PCD and ME are supported by Marie Curie Career Integration Grant Nos. PCIG12-GA2012-333990 and PCIG12-GA-2012-334056 within the Seventh Framework Programme of the European Union. The authors also acknowledge support by grants from the Medical Research Council, the Royal College of Surgeons of England, the Sparks Children Medical Charity, the British Foreign Office for the UK/USA Stem Cell Collaboration Award and the Mittal Research Fund. PDC is supported by Great Ormond Street Hospital Childrens Charity. We would also like to thank the Royal Society/Wolfson Foundation for the tissue engineering laboratory refurbishment grant obtained for the Pediatric Surgery Department at University College London (UCL) Institute of Child Health. Authors thank the ESRF (Grenoble, France) synchrotron for having provided beamtime and the ID17 staff for their support in image acquisition.

\section{REFERENCES}

[1] Murphy, S. and Atala, A., "Organ engineering combining stem cells, biomaterials, and bioreactors to reproduce bioengineered organs for transplantation," Bioessays 35, 163-72 (2013).

[2] Guyette, J., Gilpin, S., Charest, J., Tapias, L., Ren, X., and Ott, H., "Perfusion decellularization of whole organs," Nature Protoc. 9, 1451-68 (2014).

[3] Totonelli, G., Maghsoudlou, P., Garriboli, M., Riegler, J., Orlando, G., Burns, A., Sebire, N., Smith, V., Fishman, J., Ghionzoli, M., Turmaine, M., Birchall, M., Atala, A., Soker, S., Lythgoe, M., Seifalian, A., Pierro, A., Eaton, S., and Coppi, P. D., "A rat decellularized small bowel scaffold that preserves the villus-crypt architecture for intestinal regeneration," Biomaterials 33, 3401-10 (2012).

[4] Appel, A., Anastasio, M., Larson, J., and Brey, E., "Imaging challenges in biomaterials and tissue engineering," Biomaterials 34, 6615-30 (2013). 
[5] Bravin, A., Coan, P., and Suortti, P., "X-ray phase-contrast imaging: from pre-clinical applications towards clinics," Phys. Med. Biol. 58, R1-R35 (2013).

[6] Hagen, C., Diemoz, P., Endrizzi, M., Rigon, L., Dreossi, D., Arfelli, F., Lopez, F., Longo, R., and Olivo, A., "Theory and preliminary experimental verification of quantitative edge illumination x-ray phase contrast tomography," Opt. Express 22, 7989-8000 (2014).

[7] Pfeiffer, F., Weitkamp, T., Bunk, O., and David, C., "Phase retrieval and differential phase-contrast imaging with low-brilliance x-ray sources," Nature Phys. 2, 258-61 (2006).

[8] Olivo, A. and Speller, R., "A coded-aperture technique allowing x-ray phase contrast imaging with conventional sources," Appl. Phys. Lett. 91, 074106 (2007).

[9] Hagen, C., Maghsoudlou, P., Totonelli, G., Diemoz, P., Endrizzi, M., Rigon, L., Menk, R., Arfelli, F., Dreossi, D., Brun, E., Coan, P., Bravin, A., Coppi, P. D., and Olivo, A., "High contrast microstructural visualization of natural acellular matrices by means of phase-based x-ray tomography," Sci. Rep. 5, 18156 (2015).

[10] Olivo, A., Arfelli, F., Cantatore, G., Longo, R., Menk, R., Pani, S., Prest, M., Poropat, P., Rigon, L., Tromba, G., Vallazza, E., and Castelli, E., "An innovative digital imaging set-up allowing a low-dose approach to phase contrast applications in the medical field," Med. Phys. 28, 1610-9 (2001).

[11] Munro, P., Hagen, C., Szafraniec, M., and Olivo, A., "A simplified approach to quantitative coded aperture x-ray phase imaging," Opt. Express 9, 11187-201 (2013).

[12] Diemoz, P., Endrizzi, M., Zapata, C., Pesic, Z., Rau, C., Bravin, A., Robinson, I., and Olivo, A., "X-ray phase-contrast imaging with nanoradian angular resolution," Phys. Rev. Lett. 110, 138105 (2013).

[13] Totonelli, G., Maghsoudlou, P., Georgiades, F., Garriboli, M., Koshy, K., Turmaine, M., Ashworth, M., Sebire, N., Pierro, A., Eaton, S., and Coppi, P. D., "Detergent enzymatic treatment for the development of a natural acellular matrix for oesophageal regeneration," Pediatr. Surg. Int. 29, 87-95 (2013).

[14] Diemoz, P., Vittoria, F., and Olivo, A., "Spatial resolution of edge illumination x-ray phase-contrast imaging," Opt. Express 22, 15514-29 (2014).

[15] Zamir, A., Endrizzi, M., Hagen, C., Vittoria, F., Urbani, L., Coppi, P. D., and Olivo, A., "Robust phase retrieval for high resolution edge illumination x-ray phase contrast computed tomography in non-ideal environments," Sci. Rep. (in press).

[16] Wang, M., Zhu, P., Zhang, K., Hu, X., Huang, W., Cen, Y., Yuan, Q., Yu, X., and Wang, J., "A new method to extract angle of refraction in diffraction enhanced imaging computed tomography," J. Phys. D: Appl. Phys. 40, 6917-21 (2007).

[17] Zhu, P., Zhang, K., Wang, Z., Liu, Y., Wu, X., McDonald, S., Marone, F., and Stampanoni, M., "Low-dose, simple, and fast grating-based x-ray phase-contrast imaging," Proc. Natl. Acad. Sci. U.S.A. 107, 13576-81 (2010).

[18] Hagen, C., Endrizzi, M., Diemoz, P., and Olivo, A., "Reverse projection retrieval in edge illumination x-ray phase contrast computed tomography," J. Phys. D: Appl. Phys. 49, 255501 (2016).

[19] Zhao, Y., Brun, E., Coan, P., Huang, Z., Sztrokay, A., Diemoz, P., Liebhardt, S., Mittone, A., Gasilov, S., Miao, J., and Bravin, A., "High-resolution, low-dose phase contrast x-ray tomography for 3d diagnosis of human breast cancers," Proc. Natl. Acad. Sci. USA 109, 18290-4 (2012).

[20] Olivo, A., Gkoumas, S., Endrizzi, M., Hagen, C., Szafraniec, M., , Diemoz, P., Munro, P., Ignatyev, K., Johnson, B., Horrocks, J., Vinnicombe, S., Jones, J., and Speller, R., "Low-dose phase contrast mammography with conventional x-ray sources," Med. Phys. 40, 090701 (2013).

[21] Diemoz, P., Vittoria, F., Hagen, C., Endrizzi, M., Coan, P., Brun, E., Wagner, U., Rau, C., Robinson, I., Bravin, A., and Olivo, A., "Single-image phase retrieval using an edge illumination x-ray phase-contrast imaging setup," J. Synchrotron Radiat. 22, 1072-7 (2015). 